\title{
USING NEURAL NETWORKS FOR DETERMINING VELOCITY VECTORS OF AIR FLOW VISUALIZED BY HELIUM BUBBLES
}

\author{
Jiří Štastný ${ }^{1}$, Jan Richter ${ }^{2}$, Petr Štastný ${ }^{2}$ \\ ${ }^{1}$ Department of Informatics, Faculty of Business and Economics, Mendel University in Brno, Zemědělská 1, 61300 \\ Brno, Czech Republic \\ ${ }^{2}$ Institute of Automation and Computer Science, Faculty of Mechanical Engineering, Brno University \\ of Technology, Technická 2896/2, 61669 Brno, Czech Republic
}

\begin{abstract}
ŠŤASTNÝ JIŘÍ, RICHTER JAN, ŠŤASTNÝ PETR. 2014. Using Neural Networks for Determining Velocity Vectors of Air Flow Visualized by Helium Bubbles. Acta Universitatis Agriculturae et Silviculturae Mendelianae Brunensis, 62(4): 757-768.

One of the important characteristics of air flow is the velocity of flow. To determine the speed, in addition to other methods, we also use the helium bubbles seeding visualization method, when bubbles are injected into the air stream so that the air flow is obvious to the eye. If a video of such flow is taken, it is possible to determine velocity vectors in a pair of consecutive frames of this video footage, derived from the change in position of individual bubbles.

This article describes a method of monitoring the bubbles in consecutive video frames. During this process, helium bubbles are detected in the first image of the pair, either by a firmly defined procedure, or with the use of a neural network. For detected bubbles, the velocity vectors are determined according to the way in which they move, therefore, according to their location in the following frame. Another neural network then determines the velocity vector at any point of image, which will be implemented in the construction of vector maps for the first image. A vector map is used for comprehensive evaluation of air flow and thus, plays an important role in the development of ventilation and air conditioning systems.
\end{abstract}

Keywords: Helium bubbles seeding, air jet velocity, Multilayer Perceptron Neural Network, recognition, vector approximation, Particle Image Velocimetry, vector map

\section{INTRODUCTION}

One of the global trends of our time is to reduce costs of heating or air conditioning of inner spaces by maintaining or even improving thermal comfort inside, and it does not matter if this space is inside a house, factory or if it is even a car interior. One of the most important factors that influence thermal comfort is the way in which the air is distributed into the inner space. The observed properties of air flow are air jet shape, reach of the stream, density of air and, last but not least, the velocity of flow. In the development of air conditioning and ventilation systems, it is important to verify the functionality of the proposed system by measuring the aforementioned variables.
To determine the characteristics of the air flow, a number of methods is used. Some are based on the measurement of specific variables using suitably located sensors and measuring devices (anemometers, manometers, etc.).

Another group of evaluating methods are optical methods, which are based on optical phenomena caused by air flow (infrared thermometry, interferometry, etc.).

The air flow can also be examined by the use of visualization methods, the principle of which is to make colourless air flow visible (Škorpil et al., 2012; Richter, Štastný, 2012). This makes it possible to subsequently acquire detailed images and video records of visible streams using affordable cameras. 
One of the ways how to make air flow visible, is to gradually incorporate a colour substance into colourless air which forms continuous fibres, or the air is completely replaced with this substance. Such a substance may be e.g. fog, smoke, or other coloured gases. Pictures of the flow of such coloured air are used in particular for the detection of the air jet shape and reach of the stream (Richter et al., 2013; Śkorpil et al., 2012). The colour tone can also determine the current density in a particular place.

Another way how to visualize the air jet is by introducing a substance that does not form continuous fibres into the air. This means of visualization is described well e.g. in literature (Kerho, 1992). Suitable compounds of such type include soot, sparks or helium bubbles (Kerho, Breagg, 1994). This article only focuses on images of air flow visualized by helium bubbles.

Helium bubbles are produced by a helium bubble generator; they are about $1 \mathrm{~mm}$ in size, but during their flight they can deform or even perish by bursting. More information about helium bubbles can be found e.g. in the literature (Zhao et al., 1989). The following text describes some possibilities for determining the flow velocity vectors at the selected points. These points may be, for example, the space where the presence

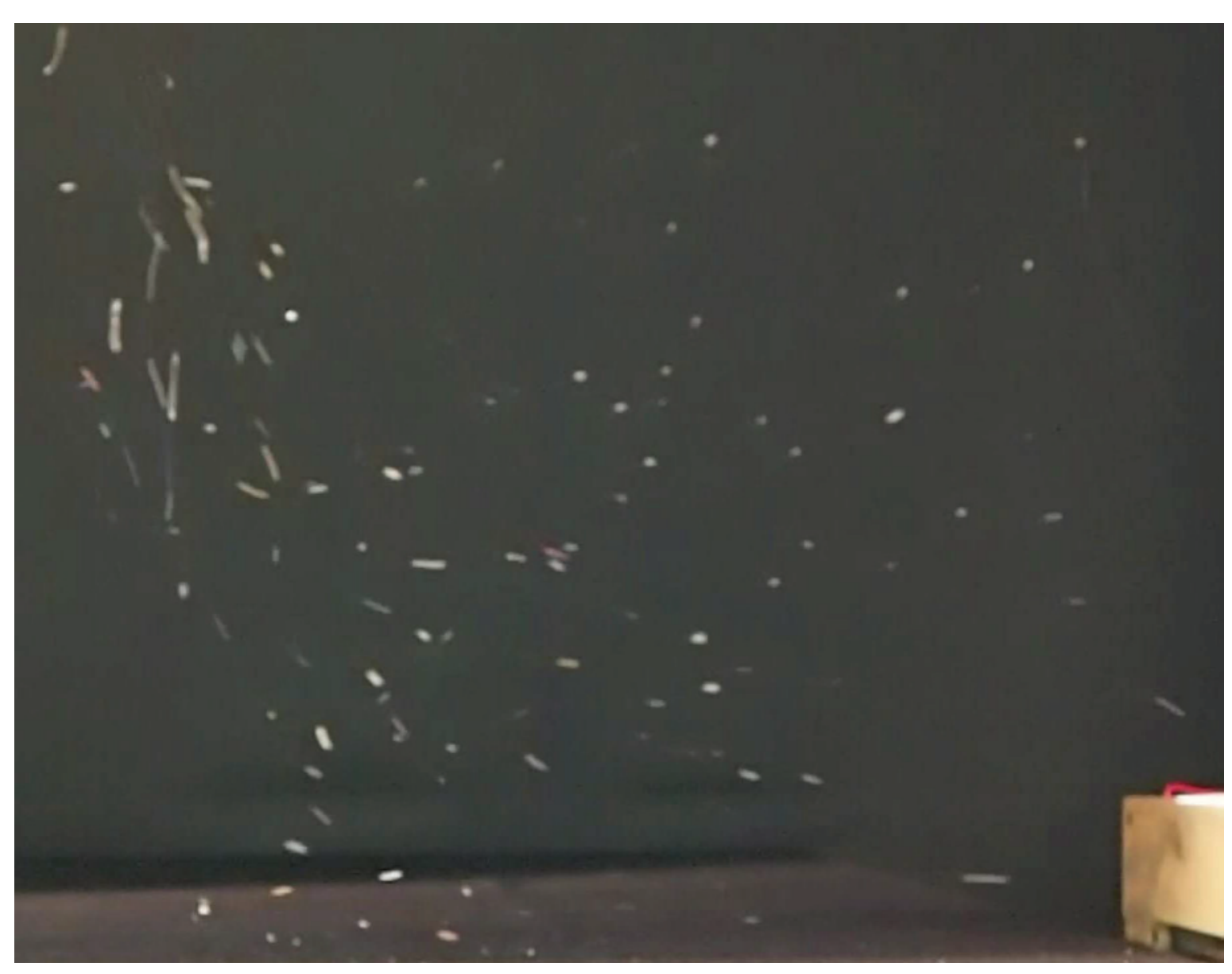

1: Image of air flow captured with longer exposure time of persons is expected, or places important for a comprehensive assessment of the flow.

To make determining of velocity possible, it is necessary to work with at least two consecutive frames. A possibility is to work even with only one image, which was, however, taken with longer exposure time. This causes the bubbles to become blurred oblong objects, the shape of which follows the trajectory of the bubble. A less clear end of the object means the position of the bubble at the beginning of the exposure period, the brighter end represents the position of the bubble at the end of exposure. Both positions and known exposure time then determine the velocity vector at the point, where the bubble was at the beginning of exposure. An example of an image shot with a long exposure time is shown in Fig. 1.

Advanced determination of velocity vectors is performed on a pair of consecutive images of record, so called frames. In this regard, there are several possible approaches of how to evaluate such pairs of images and how to obtain velocity vectors at the required points.

The first possible way is to proceed with Particle Image Velocity (PIV) analysis (Adrian, Westerweel, 2011) within which the computer evaluation runs in the way that both images are divided into subregions, where a specific vector is determined 
by cross-correlation. This vector will represent the displacement vector in the middle of the selected sub-region at a known sample time between frames.

A completely different method is the method of monitoring the movement of a specific bubble. From the known position of the bubble in the first (base) image and second (following) image and from the known sample time, the resulting direction and value of velocity is determined in the coordinates of the bubble in the base image.

The requirement of the entire evaluation is to obtain the velocity vector at any selected point. Because the velocity vectors at specific points are known, it is possible to deduce from these vectors a resulting one at any chosen point.

\section{MATERIALS AND METHODS}

Many different approaches can be used for the computer evaluation of particle motion in a stream of air. The computer analysis processes a pair of consecutive frames, which will be called the base image (basis) and following image (follower). Velocity vectors are obtained for the basis according to image of the follower.

\section{Particle Image Velocity}

PIV analysis is one of the most common ways of evaluating the motion of visible particles. The basis and follower are divided with same grid into equal sub-regions, mostly having a square shape. The size of the sub-region should depend on the density of the bubbles in the frame. If the sub-area contains a small amount of bubbles, there is a high probability that the resulting vector in the sub-region will be incorrect.

The principle of PIV evaluation is to compare corresponding sub-regions in the basis and follower. This comparison is carried out mostly with crosscorrelation using Fast Fourier Transformation, described well in (Adrian, Westerweel, 2011).

The disadvantage of PIV evaluation is the need to increase the size of the sub-regions by lowering the density of bubbles in the image. For example, if only one bubble was in the sub-area of the basis, it could happen that this bubble will no longer occur in this sub-area in the follower. The resulting vector is thus either erroneously zero (no vector is found), or another bubble accesses the area and the resulting vector is again determined incorrectly.

The sample time of the video must also be adapted to the velocity of flow. If it would not be so, the possibility increases that bubbles will not stay in the same sub-area in two consecutive frames and their actual displacement is thus not counted.

Increasing the size of sub-regions can prevent both phenomena, but it decreases the sensitivity of the method to the differences of velocity across the frame. Another way of how to increase the reliability of the method is to increase the size of the sub-area only in the ending frame. If the subarea of the basis includes the pixels in the range from $\left[X_{1}, Y_{1}\right]$ to $\left[X_{2}, Y_{2}\right]$, the corresponding sub-area of the follower will contain pixels from $\left[X_{1}-d, Y_{1}-d\right]$ to $\left[X_{2}+d, Y_{2}+d\right]$. This will reduce the possibility that the bubble will leave the investigated area. On the other hand, it raises the chance that the following area will also have bubbles, which were not in the base area. Thus, more erroneous vectors are counted, but for the contingency they have a negligible number, so the results of the Fourier transformation are not very affected. The important thing is that this adjustment will increase the weight of the correct vector of displacement.

An example of a suitable flow for PIV analysis is shown in Fig. 2. However, the mentioned conditions are very often violated for images of helium bubble flow. A typical image of a stream of helium bubbles is in Fig. 3. As you can see in this picture, the density of bubbles is much lower in comparison with Fig. 2.

Although PIV analysis exists in a variety of versions, or can even be modified into the method of particle tracking velocimetry (Adrian, Westerweel, 2011), neither of these cases do not reliably solve another undesirable phenomenon of such flow caused by working only with twodimensional images: bubbles leave the outlet with high speed, but they gradually slow down. Many of these bubbles then rise slowly in the background or foreground of the image, outside the main stream. However, according to the grid, they are located in the same area as fast bubbles in the stream. These bubbles would significantly and incorrectly affect the resulting vectors in sub-regions.

\section{Monitoring the Bubbles in Consecutive Frames}

This method tries to copy the way a human would evaluate such frames. The process consists of several steps.

\section{Bubble Detection}

In order to monitor the movement of the bubbles, the bubble should first be correctly detected and its representative should be chosen, which is the point that deputizes the bubble. Bubble detection proceeds for each pixel of the bitmap of the base image, while it is necessary to determine whether the pixel belongs to a bubble or to the background. Once a pixel of a bubble is found, then its neighbourhood pixels are recursively passed, which are appropriately assigned to the bubble.

According to the method of deciding, if the pixel belongs to the bubble or to the background, bubble detection can be implemented in various ways:

The first possible way is to use detection according to light intensity level - Because the bubbles in the image are very bright, they can be detected according to light intensity. If the brightness of the pixel surpasses the selected threshold intensity, then this pixel is a pixel in the bubbles. Its neighbourhood is then recursively searched while we find pixels with a similar intensity. A middle 


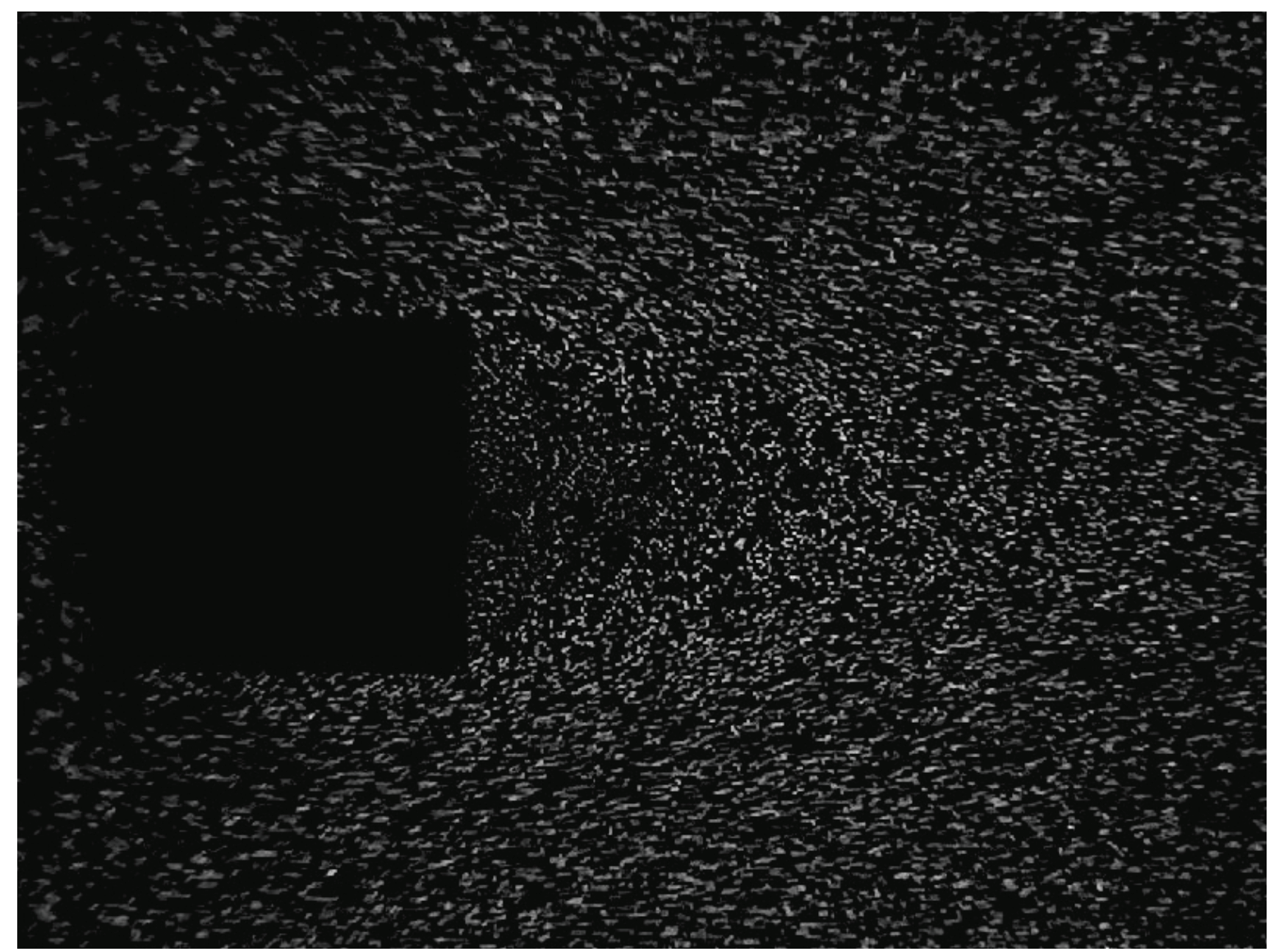

2: Image appropriate for PIV evaluation

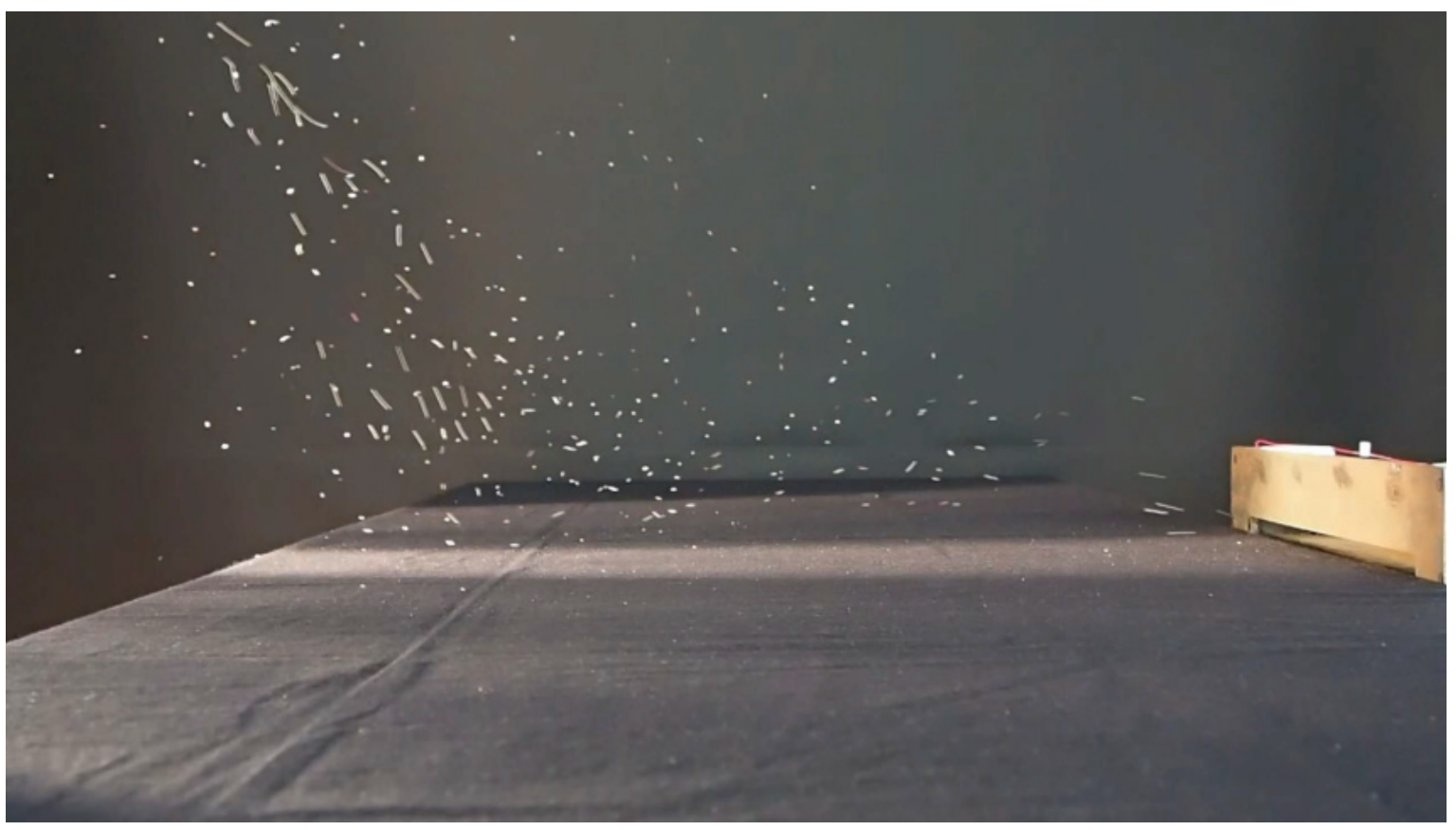

3: Typical image of air flow visualized by helium bubbles

pixel (or pixel with the highest found light intensity) will be the representative of the whole bubble.
The advantage of such means of detection rests in the time demands of procedures. With the increasing speed of movement, however, this 
bubble is more blurry and also with lower light intensity. This leads to the disadvantage of this means of detection - because of the set threshold, these very important bubbles can miss detection. On the other hand, by lowering the threshold intensity some points can be detected as bubbles incorrectly.

Another possibility is detection depending on the change of light intensity - If there is a significant reduction of the pixel intensity in the follower compared to the base image, it is very likely that the pixel belonged to bubble, which moved out of it.

The disadvantage is that this means of detection detects only bubbles that change their location. For a comprehensive evaluation, it is important to find all the bubbles, to make clear, where the bubbles are not moving any longer. Also, only pixels with noticeable light intensity decrease are detected, so the shape of the bubble can be inaccurate.

Both mentioned methods are included in the exactly defined detection - This method tries to use the positive attributes of both previously mentioned methods. Pixel bubbles are detected either by absolute intensity, or according to a fact that it has lower intensity, but this intensity decreases further in the follower (bubble left pixel coordinates). The success rate of bubble detection is thus increased.

The disadvantage of this method is that part of the bubble may not meet even one of the conditions, and therefore is not detected at all. Thus, the shape of the bubble is designated incorrectly, or the bubble can even be divided into partly detected according to level of intensity,

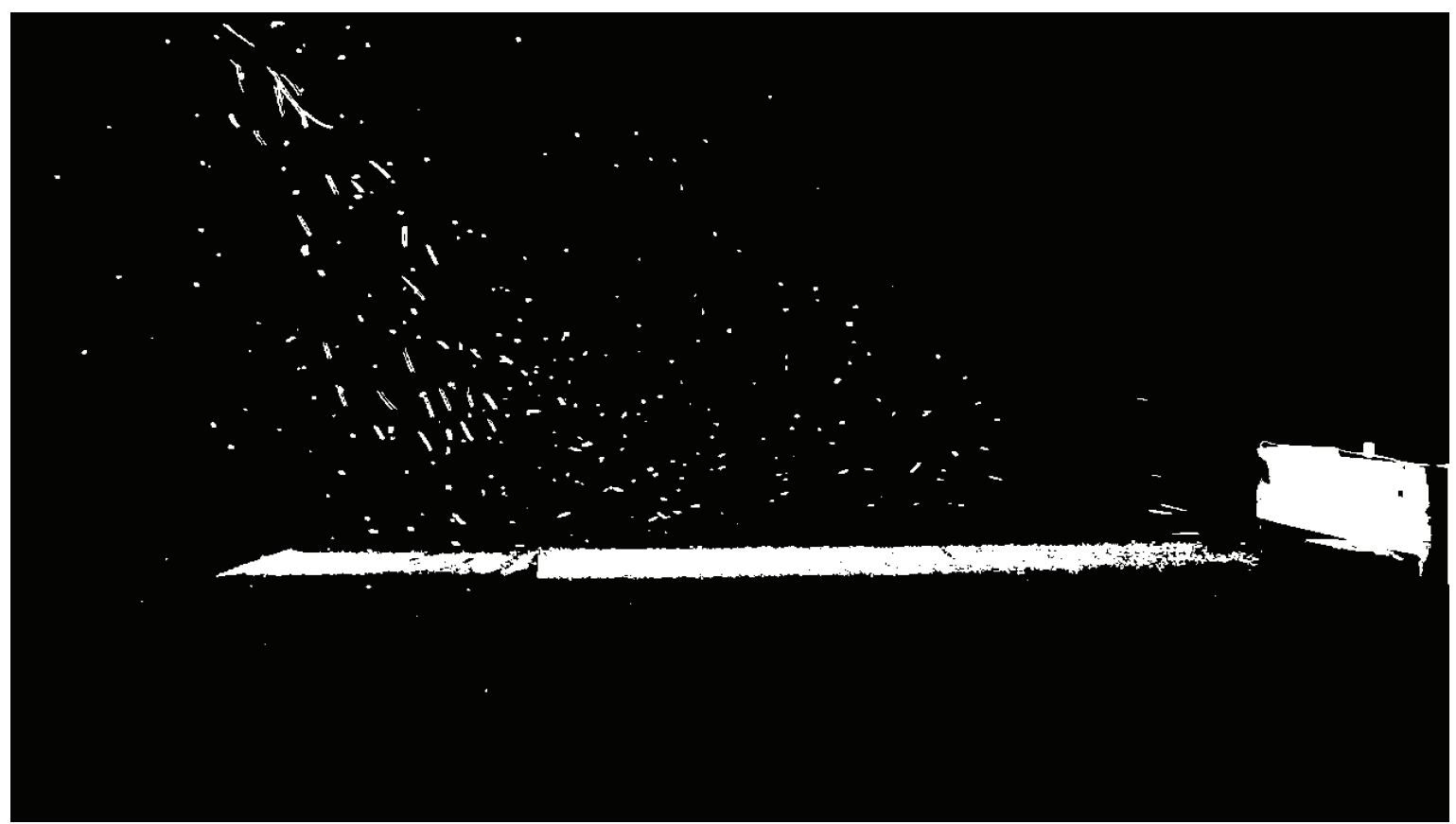

4: Figure of bubbles detected by combined exact detection and partly detected according to a change of intensity.

Another way is to use detection by means of neural network (Štencl, Štastný, 2010; Škorpil, Štastný, 2008) operates with the immediate surroundings of the examined point (e.g. $5 \times 5$ pixels). Its 25 inputs are relative light intensities of each pixel of the neighbourhood: values in the interval $\langle 0,1\rangle$, where 0 is black and 1 is white. A network will learn according to several patterns how the neighbourhood of the bubble pixel looks like and how it does not. This knowledge can then be applied - to decide whether the pixel belongs to a bubble or not. More about this evaluation method can be found in the literature (Richter et al., 2013). After finding the point of the bubble, we also recursively search and investigate its neighbourhood, thereby, the bubble is assembled. Its middle point will represent the whole bubble.

Along with the exactly defined combined detection method, detection using neural networks is the best way of how to detect bubbles. The disadvantage of this detection method is the necessity to teach the network the evaluation on patterns determined by a human for some picture captured in the same environment.

It is therefore advisable to use either the exactly defined combined detection or detection using neural networks for the detection of bubbles. Both are equivalent with regard to the success of detection, which is evident from images 3, 4, 5. Fig. 3 is a base image of the pair, where the bubbles are detected (exactly defined combined detection also uses the follower). Fig. 4 shows the result of the exactly defined combined detection of Fig. 3. neural network - A Multilayer Perceptron (MLP) 


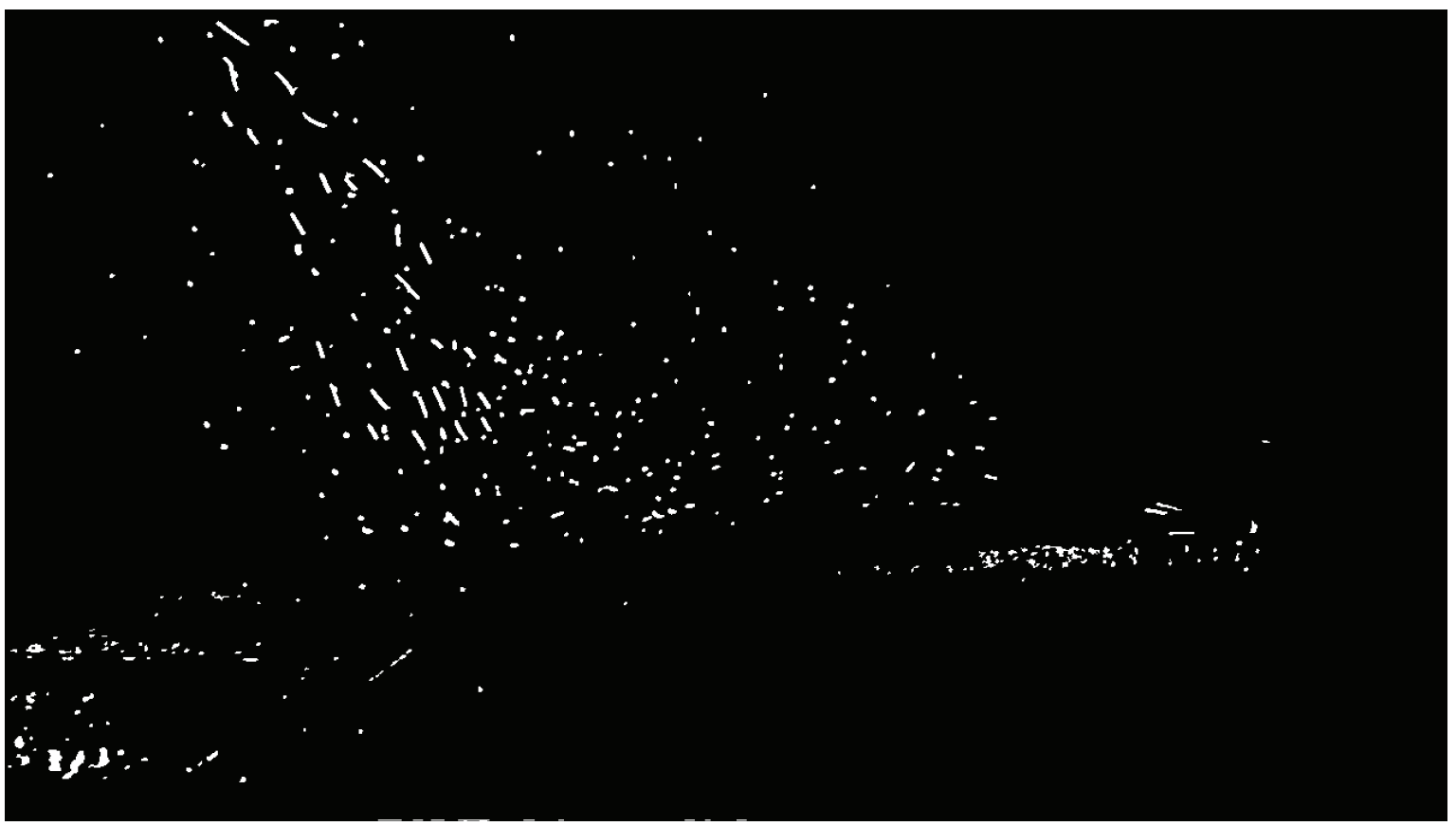

5: Figure of bubbles detected by the neural network after size limitation

For further evaluation, it is preferred to limit the bubbles in size (e.g. the maximum number of pixels of one bubble $=200$, the minimum number of pixels of one bubble $=5$ ). In this way it would be possible to remove from the list of bubbles e.g. an erroneously detected light table, suction devices (the object on the right side of the image) or dirt on the table. This treatment mainly causes a time saving; a velocity vector for these incorrect bubbles would be finally determined as zero.

Fig. 5 illustrates bubble detection of Fig. 3 with neural networks after the application of the size limit of bubbles. The neural network can generally detect a slightly larger amount of bubbles, but along with that increase, the quantity of objects incorrectly considered as bubbles.

\section{Determination of Bubble Velocity}

When bubbles are detected in the base image, it is necessary for each one to find the same bubble in the following frame. Both of these positions determine a velocity vector, located in the bubble's representative in the base image.

Since the bubble has a similar shape and also turns around its axis only minimally in the consecutive frames, it is advantageous to recognize it in the following image by its matrix of light intensities. During a construction of a matrix of intensities is first necessary to determine the dimensions of the current bubble. This will be achieved just by recursive searching according to the criteria used for the detection of bubbles. The points of the bubble lead to obtaining four coordinate extremes $X_{\min }, X_{\max }, Y_{\min }, Y_{\max }$. From the lows, we subtract one more pixel; to the highs, we add one pixel. This expansion of the area under consideration is performed so that it would also be evident from the matrix where the bubble ends and where it begins; thus, bubbles are no longer in pixels of the enlarged extremes. All pixels in the range of new extremes from $\left[X_{\min }, Y_{\min }\right]$ to $\left[X_{\max }, Y_{\max }\right]$ are then put into the matrix, which represents the look of the bubble in the base image. In addition to this matrix, the relative position of the bubble's representative in the matrix is remembered.

The principle of monitoring the bubbles is in searching for the same bubble in the following image. Therefore, the surroundings of the bubble's original position in the follower is searched. Individual pixels in this area will be regarded as representatives of the matrices. For each of them, a corresponding matrix is obtained (the position of a representative in the matrix is known as well as the size of the matrix). The light intensities of this matrix in the follower are compared with light intensities of the original bubble matrix in the base image. The aim of the whole comparison is to determine the value $d_{M}$ in the interval $\langle 0,1\rangle$, which tells us how much the original matrix and matrix in the follower differ.

$$
d_{M}=\frac{\sum_{i=1}^{m} \sum_{j=1}^{n}\left|M_{i j}-N_{i j}\right|}{255 \times m \times n},
$$

where

$d M$.... difference of the bubble's matrix in the base image and matrix in the following image,

$M$..... bubble's matrix - in the base image,

$N$........investigated matrix - in the following image, $m, n$... size of both matrices $M, N$. 
For identical matrices, $d_{M}=0$. If e.g. matrix $M$ has all pixels black and matrix $N$ has all pixels white, then $d_{M}=1$.

For the entire surroundings of a bubble, a matrix of differences $D$ is assembled in this way:

$$
D=\left[\begin{array}{ccc}
d_{M_{11}} & \cdots & d_{M_{1 q}} \\
\vdots & \ddots & \vdots \\
d_{M_{r 1}} & \cdots & d_{M_{r q}}
\end{array}\right],
$$

where

$r, q \ldots . .$. size of the bubble's neighbourhood. It is recommended to choose $r=q$ and to situate the area's location in a way that the base bubble's representative would be at the centre.

The most similar matrix can be selected as the target matrix, but it is possible that there are more similar matrices in the neighbourhood, so in addition to considering similarity, it is useful to also investigate the distance from the initial position of the base representative. The following representative will be the closest representative, the matrix of which is also similar to the base matrix of the bubble.

Matrix $D$ is used to find the representative of the follower with the minimum value of difference and also all others that have their difference close to the value of the best one; therefore, they are subject to the condition:

$$
d_{M_{i}} \leq d_{M_{\min }}(1+T),
$$

where

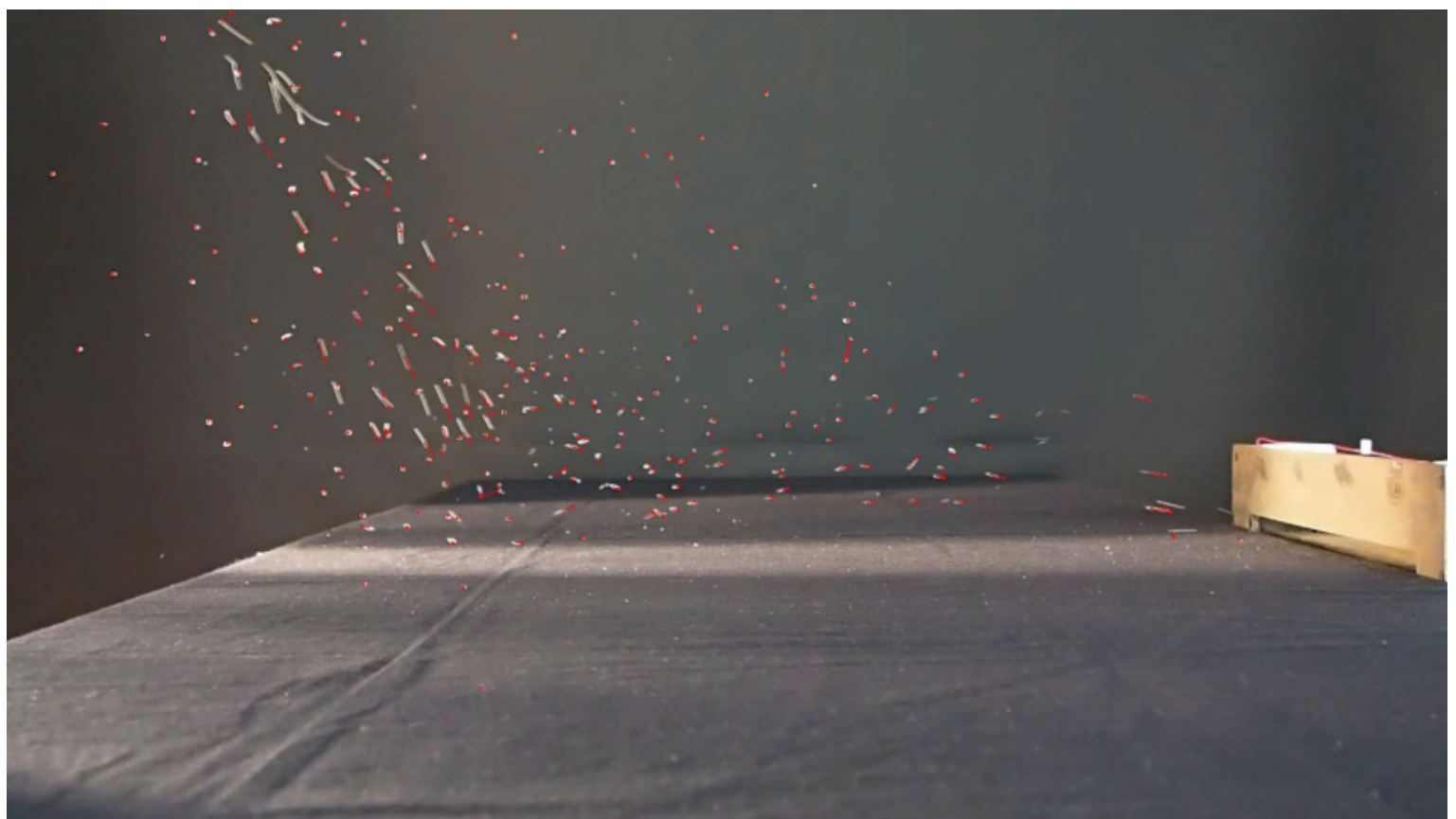

6: Figure of obtained vectors for the detected bubbles
T........tolerance of difference of the base and following matrix. Experimentally set to 0.3 .

From the selected subset of the following representative, which have matrices similar to the base bubble matrix, the one is chosen that has the lowest value of the parity criterion:

$$
K_{s}=\sqrt{\left(r_{x_{b}}-r_{x_{f j}}\right)^{2}+\left(r_{y_{b}}-r_{y_{i j}}\right)^{2}} \times d_{M_{i j}}^{s},
$$

where

$s$........factor of the preference of the similarity criterion against the distance criterion. Experimentally set to 0.25 . Because all members of the difference matrix $d_{M i}<=0$, an exponent below 1 favours the criterion of similarity.

The representative with the best value of the parity criterion is chosen as the ending point in the following image, into which the representative moves from the base location during sample time. The vector of displacement is then determined by the relative position of the two representatives:

$$
\overrightarrow{v_{d}}=r_{f}[X, Y]-r_{b}[X, Y],
$$

where

$r_{f}$........coordinates of the chosen representative in the following image,

$r_{b}$........ base coordinates of the bubble's representative.

The mentioned proceeding is applied for every detected bubble. The result of determining velocity vectors for bubbles is seen in Fig. 6 .

Comment: Exactly defined combined bubble detection was used while evaluating Fig. 6. 
As seen in the picture, not all vectors were detected correctly. The correct vector was determined for about $90 \%$ of bubbles. The incorrect vector will have a low weight in locations with a high density of bubbles. In locations with few bubbles, the incorrect vector should have a much higher weight, but there is also a much lower chance that the vector will be incorrect, because there are just few bubbles with which the right bubble could be swapped.

\section{Separation of Invalid Vectors}

As was said, in images of rapid flow of helium bubbles, a phenomenon occurs often when previously fired bubbles rise in the background or in the foreground of the frame, whereas fast bubbles in the main stream have similar $X, Y$ coordinates ( $Z$ is missing in the $2 \mathrm{D}$ image). These slow bubbles have no importance for flow evaluation, but it would distort the resulting velocity vectors in the vicinity.

There is also an opposite problem with incorrectly detected high velocity vectors, which could occur in the area where the flow has very low velocity.

For this reason, the separation of invalid vectors is carried out. The separation has two steps:

- First, it is necessary to separate the incorrectly determined vectors. If all vectors are much lower than the bubble vector in the bubble's neighbourhood, it means that such a vector is in this area incorrectly. So if there is more than one other bubble in the investigated bubble's neighbourhood and if the following condition is valid for every bubble vector in this neighbourhood, the vector of the investigated bubble is invalid.

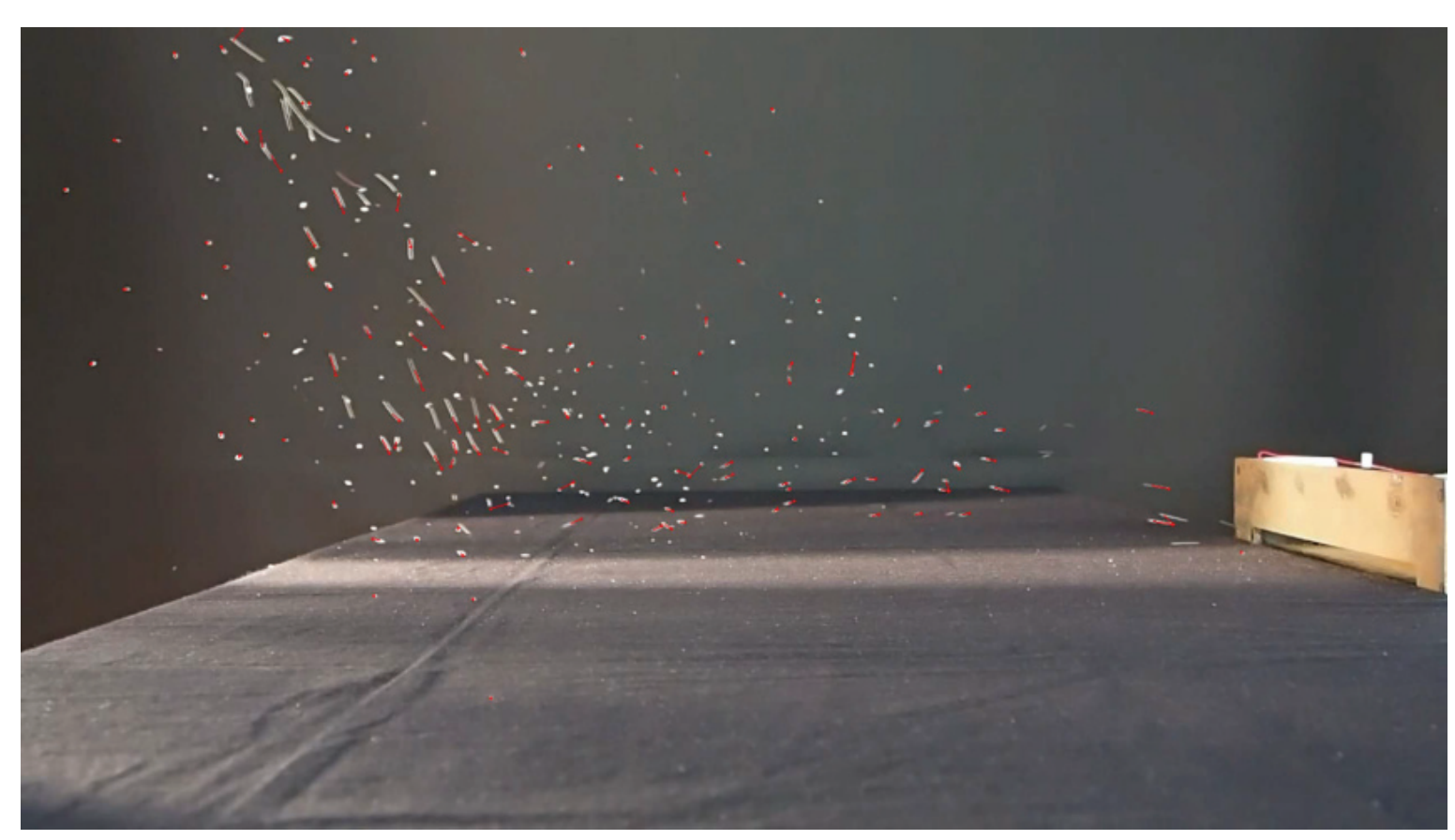

7: Result of invalid vector separation

$$
\left|\overrightarrow{v_{b}}\right|>k \times\left|\overrightarrow{v_{i}}\right|+q
$$

where

$v_{b}$.........velocity vector of the investigated bubble,

$v_{i}$.........vector of the bubble in the neighbourhood of the investigated one,

$k$.........tolerance of vector size (e.g. 2)

q..........constant of difference. Prevents deleting valid zero vectors (e.g. 3).

- The second step is to separate the unimportant vectors. In the resulting list, we will include only those bubble vectors that do not have another vector with a much higher velocity in their vicinity (e.g. to a distance of 50 pixels). The vector is separated if vector $v_{i}$ exists in its neighbourhood, satisfying the condition:

$$
\left|\overrightarrow{v_{i}}\right|>k \times\left|\overrightarrow{v_{b}}\right|+q,
$$

where

$v_{b}$.........velocity vector of the investigated bubble,

$v_{i}$........vector of the bubble in the neighbourhood of the investigated one,

$k$.........tolerance of vector size (for example 2),

q..........constant of difference. Prevents deleting valid zero vectors (for example 3 ).

As can be seen, both steps use the same condition, but for opposite purposes. The result of the separation of invalid vectors is shown in Fig. 7. The phenomenon occurs when bubble vectors with low velocity do not exist in the vicinity of bubbles with high velocity vectors.

\section{Determining the Velocity of Flow at the Chosen Point}

Previous sections have led to obtain vectors at specific points, where representatives of bubbles 
were located in the base frame. These vectors should be used to derive the velocity vector at a point, where we need to know it. For this purpose, the MLP neural network can again be very effectively used (Štastný, Škorpil, 2007).

The size and direction of the velocity in the image varies greatly, so the neural network would have to be extremely large to learn a whole field of vectors. Also, the number of network learning cycles would have to be much higher. However, the neural network may be used more effectively, when the solution to this problem is replicated by a human.

If a human has to determine the velocity vector at some point, he or she derives it from the next few vectors and more distant vectors will probably be completely ignored. This process can be automated by a computer if we replace a human with artificial neural networks.

When we want to determine the vector at point $\left[X, Y_{d}\right]$, we choose from a list of up to ten closest representatives of bubbles, which are also not very distant from the point $\left[X_{a}, Y_{a}\right]$ (e.g. $d_{\max }=80$ pixels). If there was no bubble in such a distance, the determination process would not be done at all and the velocity at point $\left[X_{a}, Y_{a}\right]$ was evaluated as zero. If there is only one bubble at a close distance, the value of the resulting vector will be determined as follows:

$$
d X_{a}=1-\frac{d X_{b}}{d_{\max }}
$$

where

$d X_{a}$... coordinate $X$ of the vector located at $\left[X_{a}, Y_{a}\right]$,

$d X_{b} \ldots$ coordinate $X$ of the velocity vector of the only close bubble.

Similar proceedings would be done for the vector coordinate $Y$. It means that the value of only the selected vector will have a value of 1 when the wanted vector is located directly at the bubble's representative, and a value of 0 when it reaches the border of the defined close area.

In other cases, representatives of selected bubbles are passed to the neural network as a pattern of inputs and their vectors as a pattern of outputs.

Inputs can be passed as relative positions in the bitmap of the base image; however, for better accuracy, it is advantageous to pass them as relative positions in the neighbourhood of point $\left[X_{a}, Y_{a}\right]$. Since the lowest possible coordinate of the selected bubble's representative is $X_{a}-d_{\max }$ and the highest one is $X_{a}+d_{\max }$, the interval $\left\langle X_{a}-d_{\max }, X_{a}+d_{\max }\right\rangle$ is transformed into an interval $\langle 0,1\rangle$, the coordinate of representative $X_{r}$ is then transformed with this formula:

$$
X_{n}=\frac{X_{r}-\left(X_{a}-d_{\max }\right)}{2 \times d_{\max }},
$$

where
$X_{n}$......relative coordinate $X$ of the close representative transformed into the interval $\langle 0,1\rangle$,

$X_{r}$.......original absolute coordinate $X$ of the close representative,

$X_{a}$.......absolute coordinate $X$ of the point, where the velocity should be determined,

$d_{\max } \ldots$ value of the maximum distance of the close representative from point $\left[X_{a}, Y_{a}\right]$.

The coordinate $Y$ will be transformed in a similar way.

Both outputs (vectors of selected bubbles) should be transformed into the interval $\langle 0,1\rangle$ as well. Before that, it is necessary to pass vectors and find the highest change of coordinates $v_{\max }$. E.g. for vectors [1, 2], [-3, -2], [-2, -4] is $v_{\max }=4$.

This $v_{\max }$ is then multiplied, for example with 1.5, to give the network a chance to determine an even higher coordinate that was found. Coordinates $d X_{r}$ of bubble vectors will be transformed with this formula:

$$
d X_{n}=\frac{0.5}{v_{\max }} \times d X_{r}+0.5
$$

where

$d X_{n}$...relative coordinate $X$ of the velocity vector transformed into the interval $\langle 0,1\rangle$,

$d X_{r} \ldots$ original absolute coordinate $X$ of the velocity vector,

$v_{\max }$... highest possible vector coordinate.

The coordinate $Y$ of the vector will be transformed in a similar way.

So, the neural network has two input neurons and two output neurons. The hidden layer is sufficient as one with 25 neurons. Increasing the number of neurons or the use of additional layers did not prove the improvement of results.

The neural network then goes through a cycle of learning the patterns (1000 repetitions is enough). Then the neural network is able to decide the velocity vector at point $\left[X_{a}, Y_{a}\right]$, coordinates of which are passed to the network in a transformed form as $[0.5,0.5]$. Obtained results are transformed again with an inverse vector transformation:

$$
d X_{a}=\frac{\left(d X_{n}-0.5\right) \times v_{\max }}{0.5}
$$

where

$d X_{n}$...relative coordinate $X$ of the velocity vector the first output of the network,

$d X_{a} \ldots$ absolute coordinate $X$ of the resulting velocity vector.

The second output is used in a similar way for obtaining the second coordinate of vector $d Y_{a}$.

\section{Clarification of the Velocity Vectors}

To further refine the vector map, it is advantageous to repeat the entire procedure described previously for another pair of consecutive frames of recorded flow in order to determine a vector for the same 
coordinates in several different base images of the same air flow. Vectors are then simply averaged to make a resulting vector more credible.
This procedure is used not only to clarify vectors, but especially for eliminating the errors that may

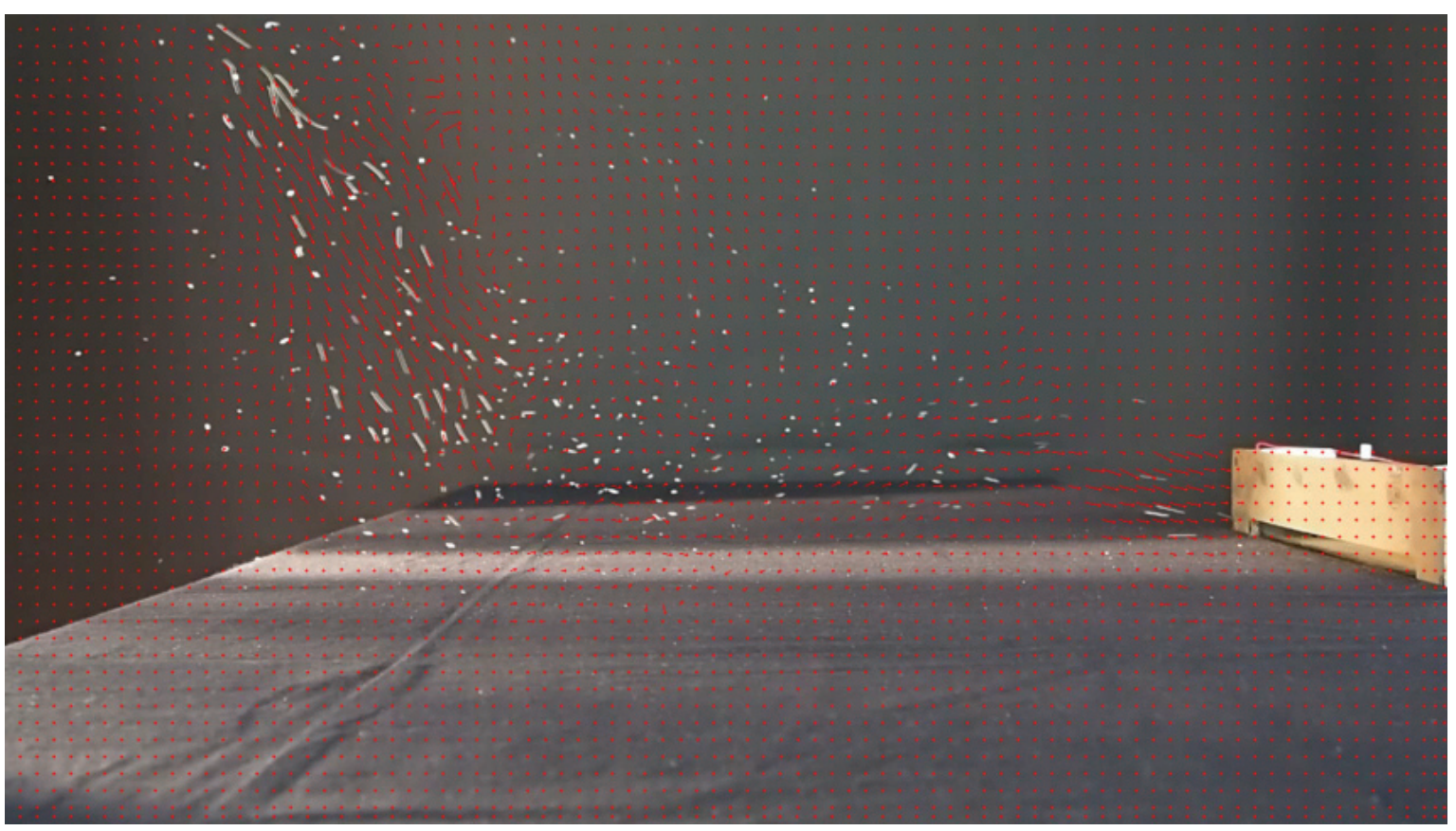

8: Map of vectors

I: Example of resulting velocity vectors for grid pixels

\begin{tabular}{|c|c|c|c|c|c|c|c|}
\hline$X$ & $\mathbf{Y}$ & $\mathrm{dX}$ & $d Y$ & $X$ & $\mathbf{Y}$ & $\mathrm{dX}$ & $d Y$ \\
\hline 299 & 14 & 0 & -2 & 314 & 14 & 0 & -2 \\
\hline 299 & 29 & -9 & -7 & 314 & 29 & -5 & -5 \\
\hline 299 & 44 & & 0 & 314 & 44 & -2 & \\
\hline 299 & 59 & -4 & 0 & 314 & 59 & -3 & 0 \\
\hline 299 & 74 & -4 & 2 & 314 & 74 & -5 & 0 \\
\hline 299 & 89 & -4 & & 314 & 89 & -3 & -5 \\
\hline 299 & 104 & 3 & 9 & 314 & 104 & -5 & -6 \\
\hline 299 & 119 & 1 & 5 & 314 & 119 & -6 & -7 \\
\hline 299 & 134 & 3 & 6 & 314 & 134 & & 3 \\
\hline 299 & 149 & 8 & 12 & 314 & 149 & 2 & 9 \\
\hline 299 & 164 & 6 & 10 & 314 & 164 & 5 & 7 \\
\hline 299 & 179 & 3 & 7 & 314 & 179 & 6 & 11 \\
\hline 299 & 194 & 6 & 9 & 314 & 194 & 8 & 14 \\
\hline 299 & 209 & 8 & 13 & 314 & 209 & 8 & 13 \\
\hline 299 & 224 & 6 & 9 & 314 & 224 & 9 & 13 \\
\hline 299 & 239 & 5 & 9 & 314 & 239 & 6 & 10 \\
\hline 299 & 254 & 7 & 10 & 314 & 254 & 7 & 11 \\
\hline 299 & 269 & 5 & 9 & 314 & 269 & 7 & 11 \\
\hline 299 & 284 & 6 & 12 & 314 & 284 & 4 & 8 \\
\hline 299 & 299 & 4 & 9 & 314 & 299 & 6 & 10 \\
\hline 299 & 314 & 2 & 8 & 314 & 314 & 5 & 11 \\
\hline 299 & 329 & 2 & 8 & 314 & 329 & 1 & 7 \\
\hline 299 & 344 & 2 & 8 & 314 & 344 & 4 & 9 \\
\hline 299 & 359 & 2 & 6 & 314 & 359 & 4 & 9 \\
\hline
\end{tabular}


occur for the determination of vectors in a single frame.

\section{RESULTS}

From the detected velocity vector at each bubble's representative, a neural network can derive the flow velocity at any point in the image. For a comprehensive evaluation of the image, a vector map should be formed for the image (the same type of result as the PIV method produces). Therefore, it is sufficient to evaluate the vectors at specific points which form a grid image.

Tab. I shows the results of the detected vectors at the selected points of the image with a original resolution of $1280 \times 720$. Plotting the resulting vector map to the original image is shown in Fig. 8. The bubbles leave the outlet in the upper left corner and fly diagonally downward. In the upper-right side of the main stream, turbulence is indicated, occurring in this area. In the middle of the figure, the bubbles slow down, but some of them are drawn into the suction device in the right part of the image.

It is important to say that there were also local inaccuracies in the evaluation. The largest of them are too large velocities just in the area of turbulence. These errors usually arise at times when the velocity vector is derived from a small number of bubble vectors, which are moreover faulty. The procedure of velocity clarification solves this issue, during which more vectors in this point are derived from different pairs of consecutive images of this flow. These velocity vectors are then averaged.

Comment: The bubbles were detected by an MLP network during the evaluation of the illustrated result.

\section{SUMMARY}

In the development of air conditioning and ventilation systems, it is important to correctly describe the flow of air which flows from the outlet into the interior. One of the most monitored characteristics is velocity in a concrete area. In addition to the practices when flow velocity is measured using sensors and measuring devices, there is also the possibility to use a visualization method for the evaluation of flow and subsequent computer processing of the air flow image. To determine the velocity, a visualization method of helium bubbles seeding into the air flow is often used. With proper illumination of the scanned area, the bubbles are clearly visible and if the video footage captures the visualized flow, it is then possible to determine the velocity vectors at any point in the image. A pair of consecutive frames is very often used for that (we called them a base image and following image). There are special methods that lead to the determination of the differences between both images, which means changes that have occurred over time, corresponding to the sample time between video frames.

The Particle Image Velocimetry evaluating method is often used for video records containing moving particles. If the stream of bubbles is not too dense, the method is not very accurate. Moreover, it is very unreliable, because the correct velocities in images are distorted by flowing bubbles in the background or foreground that randomly move and are other unimportant bubbles that are actually outside of the stream.

For these reasons, it is preferable to determine the changes in images of flow by using the method of monitoring the bubbles in consecutive frames. Its principle is to determine where the bubble moved, which was originally located at $[X, Y]$. From a known starting and ending position of the bubble, a vector of the bubble's displacement can be determined. The whole procedure for the determination of the velocity vectors at any point consists of several steps.

The first step is detection of bubbles. For this purpose, an exactly defined combined method can be used, which detects bubbles according to their light intensity and also according to their movement. Another possibility is to use a suitably learned MLP neural network as a bubble detector.

The next step is the correct identification of the bubbles in the following frame. The bubble is remembered in the base image by its matrix of light intensities, which is being compared with matrices in the immediate vicinity of the following image. The appropriate bubble is then selected by a combination of criteria of matrix similarities and criteria of distance that the bubble would travel. From a known starting and ending position of the bubble, its velocity vector is obtained.

The important action is the separation of an invalid velocity vector. Invalid vectors are vectors of bubbles that randomly fly in front or behind the investigated air flow.

The known position of bubbles and their vectors are passed to another MLP neural network, which is able to deduce from these values the velocity vector at any point in the image. By using this procedure, we can create a vector map, from which the flow characteristics are clearly visible.

The method for monitoring bubble images with a thin stream of bubbles is very effective in comparison with the PIV method. It is more accurate, it uses artificial intelligence methods and, therefore, better corresponds with the procedures that humans use for deducing the velocity vectors.

With the ability to determine the velocity vector at any point in the scanned plane, it is then possible to evaluate the characteristics of the air flow and adjust the ventilation system requirements for thermal comfort in the interior in a way to make ventilation and air conditioning effective, but not to bother people in the interior. 


\section{REFERENCES}

ADRIAN, R. J., WESTERWEEL, J. 2011. Particle image velocimetry. Cambridge University Press.

KERHO, M. 1992. A Study Of The Accuracy Of Neutrally Buoyant Bubbles Used As Flow Tracers In Air. Master's Thesis. Urbana, IL: University of IllinoisChampaign.

KERHO, M., BREAGG, M. 1994. Neutrally buoyant bubbles used as flow tracers in air. Experiments in Fluids, 16(6): 393-400. R 10.1007/BF00202064. DOI: 1007/BF00202064.

RICHTER, J., ŠŤASTNÝ, J. 2012. Fog Border Detection in the Airflow Image. In: Advances in Computer Science. Proceedings of the $6^{\text {th }}$ WSEAS European Computing Conference ECC'12. Prague, Czech Republic, 134-139.

RICHTER, J., ŠŤASTNÝ, J., JEDELSKÝ, J. 2013. Estimations of Shape and Direction of an Air Jet Using Neural Networks. In: MENDEL 2013. $19^{\text {th }}$ International Conference on Soft Computing. Brno: Brno University of Technology, 221-226.
ŠKORPIL, V., RICHTER, J., ŠŤASTNÝ, J., LÝSEK, J., SZOCZ, J. 2012. Computer Analysis of Airflow Images Visualized by Smoke. In: International Conference CSCC 2012, Recent Researches in Communication and Computers. Kos island, Greece. 89-94.

ŠKORPIL, V., ŠŤASTNÝ, J. 2008. Comparison of Learning Algorithms. In: $24^{\text {th }}$ Biennial Symposium on Communications. Kingston, Canada, 231-234.

ŠTENCL, M., ŠŤASTNYY, J. 2010. Neural Network Learning Algorithms Comparison on Numerical Prediction of Real Data. In: Proceedings of $16^{\text {th }}$ International Conference on Soft-Computing MENDEL 2010. Brno: Brno University of Technology, 280285.

ŠŤASTNÝ, J., ŠKORPIL, V. 2007. Analysis of Algorithms for Radial Basis Function Neural Network. Personal Wireless Communications, Springer, 1: 54-62.

ZHAO, D., WANG, Z., ZHANG, X., OIN, J., LANG, X. 1989. Color Helium Bubble Flow Visualization Technique. Journal Of Aircraft, 26(6): 499-502.

Jiří Štastný: jiri.stastny@mendelu.cz 